\title{
Simple stool processing method for the diagnosis of pulmonary tuberculosis using GeneXpert MTB/RIF
}

\author{
To the Editor:
}

The diagnosis of pulmonary tuberculosis (pTB) in young children often relies on clinical diagnosis because young children are usually unable to produce a sputum sample. Sputum induction or gastric aspiration can be applied to obtain a sample for microbiological diagnosis but these methods cause discomfort, stress and pain, and cannot be performed at the lowest levels of the healthcare system, thus limiting access to pTB diagnosis of children.

However, stool samples can be obtained easily and have been shown to contain Mycobacterium tuberculosis from swallowed sputum [1]. Although stool has historically received little attention as a sample to detect pTB [2], recent publications highlight its value for a bacteriological diagnosis in children and persons living with HIV [3-5]. GeneXpert MTB/RIF (Xpert) (Cepheid, Maurens-Scopont, France) has produced accurate results on stool samples of children, with a specificity and sensitivity of over $95 \%$ and $80 \%$, respectively, when compared to Xpert on respiratory samples $[6,7]$, which is the primary diagnostic test for tuberculosis [8]. However, the stool sample processing methods described so far are complex and often mirror culture processing, requiring equipment for decontamination, neutralisation and centrifugation. Some apply a commercial buffer to increase release of bacteria or flotation with sugar to concentrate the $M$. tuberculosis bacilli $[5,6]$. Recently described methods not including centrifugation still use addition of glass beads and filtration $[6,9]$. Such methods cannot be easily implemented at the lower laboratory level in low-income, high-burden countries.

Xpert testing is accessible at the lower healthcare levels where sick children mostly initially present; therefore, using it on stool samples could substantially improve access to a bacteriological diagnosis for tuberculosis in children. Here, we describe a simple processing method that is almost as simple as sputum processing for Xpert testing with potential for use at primary health care level.

This laboratory study was performed between October 1, 2016, and May 30, 2017, following the ethical standards of the Helsinki Declaration (1975), in Dr Hasan Sadikin Hospital in Bandung, Indonesia, a tertiary care hospital for pulmonary diseases. Per the standard of care, caretakers were asked for informed consent for each of the procedures and subsequent samples taken for diagnosing pTB in the children. One stool sample was collected for consecutive children under 15 years of age with presumptive pTB. Most children also submitted one respiratory sample obtained pre-prandially by gastric aspiration for children aged up to 5 years or by sputum induction using a nebuliser for older children. Respiratory samples were processed as described previously $[10,11]$.

Stool samples were processed using a simple stool processing method that approaches the procedure of processing sputum for Xpert testing: $\sim 1 \mathrm{~g}$ stool, picked from the sample using a wooden applicator stick, was added to $10 \mathrm{~mL}$ PBS of pH 7.4 (Gibco, Schwerte, Germany), mixed by vigorous shaking and left for $\geqslant 10 \mathrm{~min}$ for stool particles to gravitate, after which $2 \mathrm{~mL}$ of the supernatant was mixed with $2-4 \mathrm{~mL}$ of the Xpert MTB/Rif sample reagent provided in the Xpert kit. After $15 \mathrm{~min}, 2 \mathrm{~mL}$ of this mixture was transferred into a cartridge for Xpert testing. Inconclusive tests were repeated once with the remaining $2 \mathrm{~mL}$ of the mixture. We calculated binomial exact confidence intervals for proportions.

@ERSpublications

A simple processing method for stool with Xpert MTB/RIF yields reliable results for young children, thus providing opportunity for painless bacteriological diagnosis of TB in children at the lowest healthcare levels. http://ow.ly/8CEI30nb0He

Cite this article as: Andriyoko B, Janiar H, Kusumadewi R, et al. Simple stool processing method for the diagnosis of pulmonary tuberculosis using GeneXpert MTB/RIF. Eur Respir J 2019; 53: 1801832 [https:// doi.org/10.1183/13993003.01832-2018]. 
In total, 36 children were included in this study with a median age of 17 months (interquartile range 5.5-78 months); 20 (56\%) children were $<2$ and five (14\%) were $2-5$ years old. M. tuberculosis was detected in six (17\%) children, either on one or both samples (table 1). 29 (81\%) children also submitted one respiratory sample; for 20 (69\%) children, this was obtained by gastric aspiration. For 27 out of 29 children, a valid test result was obtained for both the stool and the respiratory sample, and for $24(89 \%$, 95\% CI 71-98\%) of these, the results were concordant (table 1). The three (8\%, 95\% CI 2-22\%) children with discordant results all tested $M$. tuberculosis positive on stool but not on respiratory samples. Their median age was 5 months (versus 11 years for children with concordant $M$. tuberculosis-positive results on stool and respiratory samples). Children with bacteriologically confirmed tuberculosis were aged between 3 months and 13 years (median 3.2 years). All children diagnosed with TB were started on antituberculous treatment.

Using a simple two-step processing method, high concordance between Xpert test results on respiratory samples and stool was obtained. We show that testing stool samples with Xpert can increase the number of children with a bacteriological confirmation of tuberculosis, with three additional tuberculosis cases detected. Possibly, these additional cases had extrapulmonary tuberculosis, such as miliary/disseminated and gastrointestinal tuberculosis, and shed more bacilli via their stool than respiratory samples. Especially in young children, the immune system is not fully mature, which may cause disseminated disease and bacteria replicating in multiple tissues. Haematogenous tuberculous dissemination has been found among patients testing positive on urinary lipoarabinomannan (LAM), which is likely due to mycobacteriuria [12]. Urine of patients testing positive on urinary LAM was also Xpert positive [13]. A similar process may be provoked in the intestines of young children with tuberculosis. Another possibility is that the respiratory samples of these children were of inferior quality. BonNAve et al. [14] obtained lower diagnostic yields from gastric aspiration (60\%) than from stool (64\%).

The rate of inconclusive test results was higher for stool samples (six out of 40 tests, 15\%) than respiratory samples (one out of $30,3 \%$ ); for two $(6 \%)$ out of 36 stool samples, repeating the test on the remaining mixture did not lead to interpretative results. Six of the seven inconclusive test results had error codes (2008, 5006 or invalid) suggesting solid particles were blocking the cartridge. Thus, there is room for further optimisation of the processing method, e.g. by providing clear instructions on every critical step in the process, from the volume of stool (by type of stool sample) to be picked, to mixing of stool and buffer, and transfer of supernatant into the Xpert cartridge.

This small study has several limitations. First of all, it included a limited number of children, of whom only six were found to be $M$. tuberculosis positive. Secondly, it was conducted in a tertiary care hospital that receives children in whom tuberculosis is highly suspected but not yet diagnosed, some of whom are severely ill. Thirdly, since this was a laboratory study, we had no influence on which children were selected for submitting stool samples nor do we know if additional children were diagnosed on clinical grounds only. In addition, we have no information about the outcome of antituberculous treatment or previous tuberculous episodes.

Although this study was performed in a tertiary care hospital, the stool processing method can safely be applied at lower healthcare levels, as no biosafety cabinet or complex, expensive or difficult-to-obtain equipment is needed, although PBS is not available everywhere in Indonesia. An even simpler and safer variant of the method presented here, the KNCV Simple One-step Stool Method for Tuberculosis

\begin{tabular}{|c|c|c|c|c|}
\hline \multirow{2}{*}{$\begin{array}{l}\text { Stool sample Xpert } \\
\text { result }\end{array}$} & \multicolumn{3}{|c|}{ Xpert result on respiratory sample } & \multirow[t]{2}{*}{ Total } \\
\hline & $\begin{array}{l}\text { M. tuberculosis } \\
\text { positive }\end{array}$ & $\begin{array}{l}\text { M. tuberculosis } \\
\text { negative }\end{array}$ & $\begin{array}{l}\text { No sample } \\
\text { available }\end{array}$ & \\
\hline M. tuberculosis positive & 3 & 3 & 0 & 6 \\
\hline M. tuberculosis negative & 0 & 21 & 7 & 28 \\
\hline Error/invalid & 0 & 2 & 0 & 2 \\
\hline Total & 3 & 26 & 7 & 36 \\
\hline
\end{tabular}

Four stool samples and one gastric aspiration sample did not yield a conclusive result on the first test. Repetition of the test resulted in two additional test results for the stool sample and one additional test result for the gastric aspiration sample. Xpert: GeneXpert MTB/RIF (Cepheid, Maurens-Scopont, France); M. tuberculosis: Mycobacterium tuberculosis. 
Detection, omits the PBS step as $\sim 1 \mathrm{~g}$ stool is directly added to the sample reagent. This method will be tested in more health facilities to demonstrate the feasibility and acceptability of routine implementation. We anticipate that such simple, noninvasive methods may radically improve the access to a bacteriological diagnosis of tuberculosis, especially in very young children.

Basti Andriyoko', Harini Janiar ${ }^{2}$, Retno Kusumadewi ${ }^{3}$, Eveline Klinkenberg ${ }^{4,5}$, Petra de Haas ${ }^{4}$ and Edine Tiemersma ${ }^{4}$

${ }^{1}$ Molecular Biology Division, Dept of Clinical Pathology, Hasan Sadikin General Hospital, Bandung, Indonesia. ${ }^{2} \mathrm{KNCV}$ TB Foundation, Indonesia office, Jakarta, Indonesia. ${ }^{3}$ National Tuberculosis Program, Jakarta, Indonesia. ${ }^{4} \mathrm{KNCV}$ Tuberculosis Foundation, The Hague, The Netherlands. ${ }^{5}$ Dept of Global Health, Amsterdam Institute for Global Health and Development, Academic Medical Centre, Amsterdam, The Netherlands.

Correspondence: Petra de Haas, KNCV Tuberculosis Foundation, PO Box 146, 2501 CC The Hague, The Netherlands. E-mail: petra.dehaas@kncvtbc.org

Received: Oct 052018 | Accepted after revision: Nov 242018

Acknowledgements: Heda Melinda Nataprawira (Pediatric Respirology Division, Dept of Child Health, Hasan Sadikin General Hospital and Faculty of Medicine, Universitas Padjadjaran, Bandung, Indonedia) is thanked for recruiting children, and Ida Parwati (Dept of Clinical Pathology, Hasan Sadikin General Hospital and Faculty of Medicine, Universitas Padjadjaran) for her supervision and support of the laboratory work for this project.

Conflict of interest: None declared.

Support statement: This work was partly funded by US Agency for International Development (USAID) through Challenge TB under the terms of Agreement No. AID-OAA-A-14-00029. The contents are the responsibility of the authors and do not necessarily reflect the views of USAID or the US Government. Funding information for this article has been deposited with the Crossref Funder Registry.

\section{References}

1 Cordova J, Shiloh R, Gilman RH, et al. Evaluation of molecular tools for detection and drug susceptibility testing of Mycobacterium tuberculosis in stool specimens from patients with pulmonary tuberculosis. J Clin Microbiol 2010; 48: 1820-1826.

2 Graham SM, Cuevas LE, Jean-Philippe P, et al. Clinical case definitions for classification of intrathoracic tuberculosis in children: an update. Clin Infect Dis 2015; 61: S179-S187.

3 Walters E, van der Zalm MM, Palmer M, et al. Xpert MTB/RIF on stool is useful for the rapid diagnosis of tuberculosis in young children with severe pulmonary disease. Pediatr Infect Dis J 2017; 36: 837-843.

4 Nicol MP, Spiers K, Workman L, et al. Xpert MTB/RIF testing of stool samples for the diagnosis of pulmonary tuberculosis in children. Clin Infect Dis 2013; 57: e18-e21.

5 Marcy O, Ung V, Goyet S, et al. Performance of Xpert MTB/RIF and alternative specimen collection methods for the diagnosis of tuberculosis in HIV-infected children. Clin Infect Dis 2016; 62: 1161-1168.

6 Banada PP, Naidoo U, Deshpande S, et al. A novel sample processing method for rapid detection of tuberculosis in the stool of pediatric patients using the Xpert MTB/RIF assay. PLoS One 2016; 11: e0151980.

7 Hasan Z, Shakoor S, Arif F, et al. Evaluation of Xpert MTB/RIF testing for rapid diagnosis of childhood pulmonary tuberculosis in children by Xpert MTB/RIF testing of stool samples in a low resource setting. BMC Res Notes 2017; 10: 473.

8 WHO. Automated real-time nucleic acid amplification technology for rapid and simultaneous detection of tuberculosis and rifampicin resistance: Xpert MTB/RIF assay for the diagnosis of pulmonary and extrapulmonary TB in adults and children. Policy update. Geneva, World Health Organization, 2013.

9 Walters E, Scott L, Nabeta P, et al. Molecular detection of Mycobacterium tuberculosis from stools in young children by use of a novel centrifugation-free processing method. J Clin Microbiol 2018; 56: e00781-18.

10 Hillemann D, Rusch-Gerdes S, Boehme C, et al. Rapid molecular detection of extrapulmonary tuberculosis by the automated GeneXpert MTB/RIF system. J Clin Microbiol 2011; 49: 1202-1205.

11 Helb D, Jones M, Story E, et al. Rapid detection of Mycobacterium tuberculosis and rifampin resistance by use of on-demand, near-patient technology. J Clin Microbiol 2010; 48: 229-237.

12 Lawn SD, Gupta-Wright A. Detection of lipoarabinomannan (LAM) in urine is indicative of disseminated TB with renal involvement in patients living with HIV and advanced immunodeficiency: evidence and implications. Trans R Soc Trop Med Hyg 2016; 110: 180-185.

13 Wood R, Racow K, Bekker LG, et al. Lipoarabinomannan in urine during tuberculosis treatment: association with host and pathogen factors and mycobacteriuria. BMC Infect Dis 2012; 12: 47.

14 Bonnave PE, Raoult D, Drancourt M. Gastric aspiration is not necessary for the diagnosis of pulmonary tuberculosis. Eur J Clin Microbiol Infect Dis 2013; 32: 569-571.

The content of this work is copyright of the authors or their employers. Design and branding are copyright 